\title{
Hypothesis-driven research for hypothesis-driven application
}

\author{
Peter Nick $^{1}$
}

Published online: 27 March 2015

(C) Springer-Verlag Wien 2015

Science is part of society not only because the funding for science usually comes from the tax payer but also because the motivation for science often originates in questions and problems posed by society. In a time where economic constraints also have reached the funding of research, scientists have to explain, why and how their work contributes to address these questions and problems.

This move towards applicability seems to weaken the position of hypothesis-driven research - for instance, the search for novel active compounds in medicine or agriculture is often conducted as high-throughput screening of compound libraries. These advances in robotics and automatisation seem to make scientific reasoning dispensible. However, this view would be superficial: good application is always based on good science. And good science requires two elements: clear concepts and a thorough knowledge of the experimental system (which in turn is based on solid, high-quality description).

The biology of cells was born from a (at that time quite daring) conceptual impulse, the cell theory, conceived by the botanist Schleiden and the animal physiologist Theodor Schwann. In the following decades, this branch of science proceeded mainly along conceptual debates on the general principles guiding the division, growth, movement, and differentiation of cells. In other words: cell biology developed as a classical hypothesis-driven clade of science. This conceptual development was strongly dependent on methodological advances mostly related to microscopy and culminated in different important

\section{Peter Nick}

peter.nick@kit.edu

1 Molecular Cell Biology, Botanical Institute, Karlsruhe Institute of Technology, Karlsruhe, Germany applications. For instance, the agricultural application of green gene technology was a direct consequence of the long endeavour to proof the cell theory also for plant cells (for review see Opatrný 2014), and the discovery of inducible pluripotent stem cells had been inspired more than 100 years ago by the attempt to find a cellular base for inheritance (Weismann 1892). Two contributions to the current issue illustrate why and how hypothesisdriven research is needed to design new fields of application.

The concise and conceptual review of Johansen et al. (2015) at first glance looks like "pure science": the question, how the division spindle propels the duplicated chromosomes towards the cell poles is general, important and still astonishingly unclear. The widely accepted Pac-Man model assumes that microtubule disassembly at the kinetochore is responsible for the poleward movement of chromosomes during anaphase, an alternative spindle matrix model assumes that the force is generated by interaction with the spindle matrix, i.e. actomyosin (for a critical review see Pickett-Heaps and Forer 2009). To discriminate between these models, optical interference has been used as classical approach; hereby, kinetochore microtubules are severed by a microbeam of UV or, more recently, by a laser beam (Forer et al. 2013). The fact that chromosomes still move on might either mean that the remaining kinetochore stubs still can link with other microtubules (that are difficult to be visualised) or it might mean that a non-microtubule matrix is responsible for the movement. The authors review classical and recent evidence on the two mechanisms and describe the drawbacks of the two modelsthey arrive at a third model, where the microtubule-nucleating protein NuMa interacts with components of the spindle matrix and thus reconciles the dynein dependency of the movement with the fact that the movement can proceed despite disruption of kinetochore microtubules. This is a clear case of hypothesis-driven research, but it has strong impact for application: if the movement is driven by the matrix, the 
actomyosin system will provide molecular targets for pharmaceutical inhibition of cell proliferation (Forer et al. 2007), which is of high relevance for the search of novel anticancer compounds.

Also, the review by Brix et al. (2015) is testing a hypothesis - that the activity of specific proteases is mainly regulated by the manner, how they passage through the secretory pathway. This means that the mechanisms, by which specific cells regulate the localisation of these enzymes into different compartments, will decide on their biological function, which can range from inducing specific differentiation events until causing cellular suicide. Using the cathepsin cysteine protease family as a model, they survey the current knowledge on intercellular trafficking and its regulation by legumain and explain how mislocalisation can contribute to specific pathologies. They also give interesting examples of how the mechanistic understanding of this regulatory trafficking can be used to design novel strategies for the control of diseases either by restoring functional traffic or by exploiting this mechanism to target deathly cargoes to cancer cells.
Conflict of interest The author declares that there is no conflict of interest.

\section{References}

Brix K, McInnes J, Al-Hashimi A, Rehders M, Tamhane T, Haugen M (2015) Proteolysis mediated by cysteine cathepsins and legumain - recent advances and cell biological challenges. Protoplasma, current issue

Forer A, Ferraro-Gideon J, Berns M (2013) Distance segregation of sex chromosomes in crane-fly spermatocytes studied using laser microbeam irradiations. Protoplasma 250:1045-1055

Forer A, Spurck T, Pickett-Heaps JD (2007) Actin and myosin inhibitors block elongation of kinetochore fibre stubs in metaphase crane-fly spermatocytes. Protoplasma 232:79-85

Johansen K, Johansen J, Forer A (2015) Movement of chromosomes with severed kinetochore microtubules. Protoplasma, current issue

Opatrný Z (2014) From Němec and Haberlandt to plant molecular biology. Plant Cell Monogr 22:1-36

Pickett-Heaps J, Forer A (2009) Mitosis: spindle evolution and the matrix model. Protoplasma 235:91-99

Weismann A (1892) Aufsätze überVererbung und verwandte biologische Fragen. Verlag von Gustav Fischer, Jena 